\title{
Catching Moving Violations using an Embedded System
}

\author{
Shivani Bhattacharjee \\ Professor, Dept of EXTC \\ D. J. Sanghvi College of \\ Engineering \\ Mumbai
}

\author{
Niramay Sanghvi \\ Student, Dept of EXTC \\ D. J. Sanghvi College of \\ Engineering \\ Mumbai
}

\author{
Varun Varadan \\ Student, Dept of EXTC \\ D. J. Sanghvi College of \\ Engineering \\ Mumbai
}

\author{
Avi Shah \\ Student, Dept of EXTC \\ D. J. Sanghvi College of Engineering \\ Mumbai
}

\author{
Aman Shah \\ Student, Dept of EXTC \\ D. J. Sanghvi College of Engineering \\ Mumbai
}

\begin{abstract}
In this paper, the aim is to design an embedded system which will efficiently assist in capturing motorists who break signals/overspeed at a monitored traffic junction. Signal breaking is a big menace in India currently, and such a system is not in place in any Indian city. Hence this system of documenting traffic violations has much potential. The design of a prototypical system using components like a Passive Infrared (PIR) sensor, a digital camera module, and a Programmable System on Chip 4 (PSoC 4) platform is outlined in the paper. The system designed will enable the penalization of those who violate traffic laws.
\end{abstract}

\section{Keywords}

Embedded system, Programmable System on Chip, Moving Violations, PIR sensors, Digital Camera Module

\section{INTRODUCTION}

A moving violation is any violation of the law committed by the driver of a vehicle while it is in motion. The term 'motion' distinguishes it from other violations such as parking violations, improper documentation, etc. Signal-jumping, also known as red light running, is a traffic violation where a motorist ignores a stop sign and "jumps" a signal anyways, i.e. they cross the signal even though they are supposed to stop. It is a major violation, accounting for 13,244 cases in the first 6 months of this year in the Indian City of Navi Mumbai, $65 \%$ more than the number of cases registered in the whole of 2013[1], causing traffic jams and a deadly threat, both for pedestrians and other motorists caught unaware by signaljumpers. In the United States as well, accidents at intersections with traffic lights account for up to $40 \%$ of all accidents and a major portion of these accidents could be attributed to motorists running a red light [2]. In this project, the aim is to design a system to detect incidences of signaljumping. A PIR motion sensor is used to detect the motion of vehicles after the signal has turned red. On detecting motion, it sends a signal to the PSoC 4. The PSoC 4, in turn activates a digital camera module which uses a Universal Asynchronous Receiver/Transmitter (UART) for communication and proceeds to take a picture of the car's license plate using it. The picture is stored in a Secure Digital (SD) card, for further uses such as evidence and documentation of moving violations. It is later used for printing a photo traffic ticket.

Furthermore, variations in the design of this project enable it to detect over-speeding by using two PIR sensors instead of one. This variation can implement an up-counter, based on which the time taken by the vehicle to travel the distance between the limits of the areas under observation of the two PIR sensors can be determined. If the value of the counter is less than a predetermined threshold value, it can be concluded that the vehicle is over-speeding, and therefore the PSoC is triggered and it makes the camera take a picture of the license plates in this case as well. This threshold value is determined using the basic formula, Time $=$ Distance/Speed, wherein the distance between the limits of the area of observation and the speed limit are taken into account.

\section{EXTERNAL COMPONENT DESCRIPTION}

\subsection{Camera Module}

The uCAM-II is a serial camera module which is attached to a host embedded system that requires a video camera or a JPEG compressed still camera [3]. The module can produce both RAW format as well as JPEG format images.

The CMOS VGA color sensor and JPEG compression chip enables the module to operate on the low power supply. The serial interface is TTL compatible and is used for connections with any host microcontroller UART or a PC COM port.

\subsubsection{Features:}

It requires a nominal supply of $5 \mathrm{~V}$ DC. The on-board serial interface is capable of transmitting JPEG still pictures or raw images up to a speed of $3.68 \mathrm{Mbps}$. It communicates with the help of a serial port. It is done with the help of a serial interface which is used by the host for communication with the UART component on the PSoC. It consists of built-in color conversion circuits for 16-bit RGB or standard JPEG images. A VGA color sensor along with a RAW/JPEG CODEC is utilized for different image resolutions

\subsubsection{Pin description}

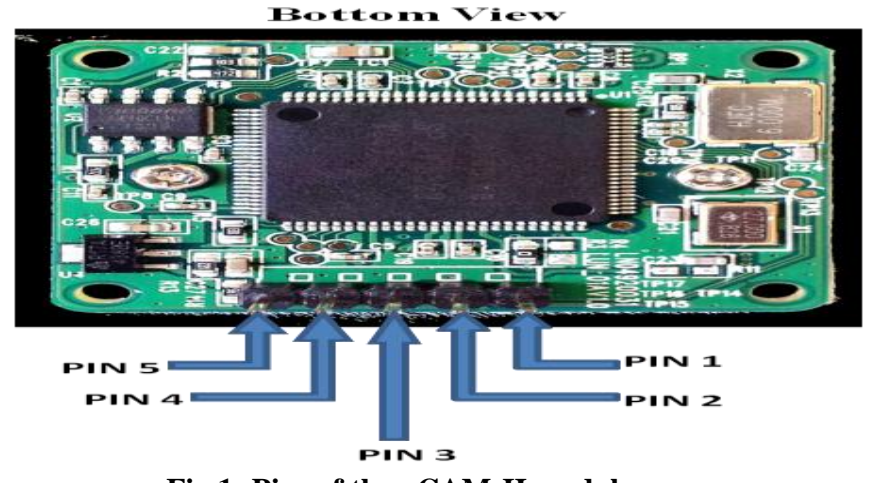

Fig 1: Pins of the uCAM-II module 
PIN 1 (+VCC): Supply input pin. This pin must be connected to a regulated supply voltage within a range of $4.5 \mathrm{~V}$ to $9.0 \mathrm{~V}$ DC.

PIN 2 (TX/Serial Transmit): It is an asynchronous serial pin. This pin must be connected to the serial receive $(\mathrm{Rx})$ pin of the UART on the PSoC. This pin works on a 3.3V logic.

PIN 3 (RX/Serial Receive): It is an asynchronous serial pin. This pin must be connected to the serial transmit (Tx) pin of the UART on the PSoC. This pin works on a 3.3V logic.

PIN 4 (GND/Ground): This pin must be connected to ground. PIN 5: Not connected

\subsection{PIR Sensor}

The HC-SR501 PIR sensor is an electronic sensor which measures infrared light radiating from objects in the field of view [4]. It does not generate any energy itself but works on the principle of detection of the infrared radiation emitted by objects. Hence, the name consists of the word "passive". It detects motion due to the change in the amount of infrared energy incident upon the sensor, in response to which the sensor sets its output pin to a logic HIGH (3.3 V) voltage

\subsubsection{Features:}

Operating voltage range: $3.6 \mathrm{~V}-20 \mathrm{~V} \mathrm{DC}$

Temperature sensitivity: Temperature compensation might be required depending upon the temperature of the body to be detected as compared to the ambient temperature. If the body is at a warmer temperature, then it becomes difficult to detect at a greater distance when the ambient temperature rises, i.e. the temperature difference becomes smaller.

It provides a high output signal of $3.3 \mathrm{~V}$ when triggered.

The quiescent current is in the microampere range $(<50 \mu \mathrm{A})$, which ensures lower power consumption when idle. This makes the sensor ideal when operated using battery power, where power conservation becomes important.

\subsubsection{Pin Description:}

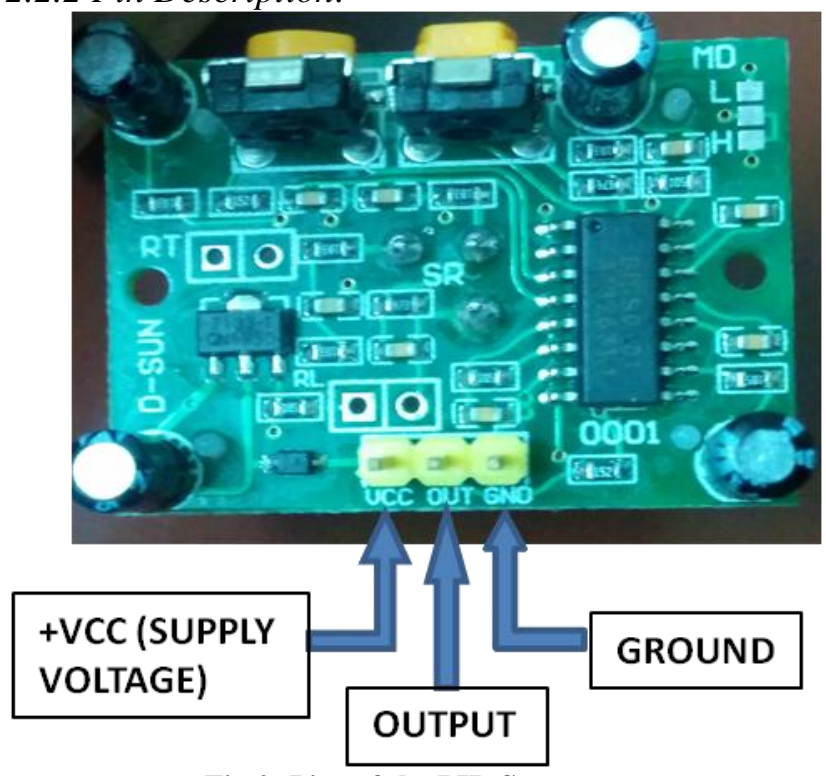

Fig 2: Pins of the PIR Sensor

VCC: This is connected to the DC supply voltage.

OUT: The output of the sensor is obtained using this pin.

GND: This pin is grounded (connected to $0 \mathrm{~V}$ )

\subsection{SD Card Breakout}

The SD card breakout socket is used to enable the access to pins on the SD card easily [5]. The pins are described as follows.

\subsubsection{Pin Description:}

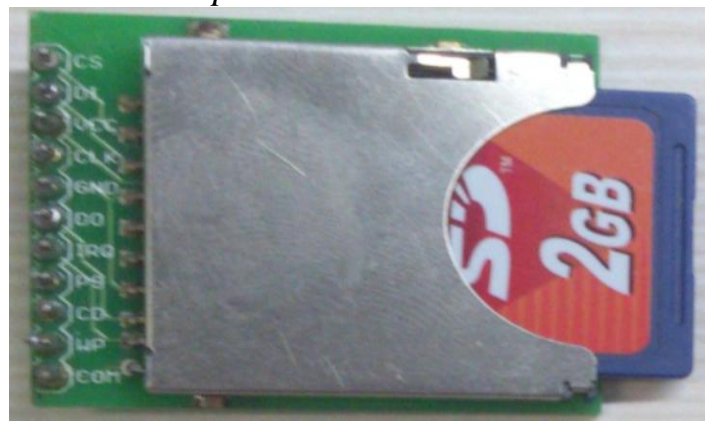

Fig 3: Pins of the SD Card Breakout

CS (Card Select): This pin allows the selection and enabling of the card.

DI (Data Input): This pin receives data from the PSoC.

VCC: This is connected to the positive supply voltage.

CLK (Clock): This is the serial clock input.

GND (Ground): This is connected to the ground

DO (Data Output): This pin sends data from the SD card to the PSoC.

IRQ \& P9: These pins are not used, instead connected to 3.3V supply using a $10 \mathrm{k} \Omega$ resistor.

CD \& WP: Neither of these pins are connected.

COM: This pin is not used; it is connected to the ground.

\section{PLATFORM USED}

PSOC is a family of microcontroller integrated circuits by Cypress Semiconductor. The Cypress architecture used in the PSoC family includes an 8-bit processor [6]. Digital as well as analog peripherals are included. The configurable analog units include comparators as well as analog-to-digital converters (ADCs). The digital units comprise of timers, counters, pulse width modulators (PWMs), Cyclic Redundancy Check (CRC) modules, a full-duplex UART, and a Serial Peripheral Interface (SPI) module. It also includes RAM and Flash memory.

PSOC 4 has been used for the project .This version of PSOC consists of the core of ARM cortex M0 microcontroller, developed by Cypress Semiconductors. [7]

PSOC Creator is a second generation software used IDE to design, debug and program various PSOC devices in general, developed by Cypress Semiconductors. The development IDE is combined with an ergonomic graphical design editor to facilitate the integration of hardware and software design. The schematic files (.cysch) enable one to easily configure various components on the PSoC, while pin connections are modified using the cydwr files. The programming is modified using the main.c file in combination with various header files.

\section{ON-BOARD COMPONENT CONFIGURATION}

The on-board PSoC components are configured as follows, in accordance to the datasheets of the various components. [8][9] UART:

Mode: Full UART.

Bits Per Second: 1/8 input clock frequency.

Parity Type: None.

Stop bits: 1.

Flow Control: None.

SDCard:

Configuration to use: Enable Full File System.

Max number of files to be opened simultaneously: 2 .

Digital Input pins:

HW Connection: Enabled.

Drive Mode: High impedance Digital. 
Initial State: Low (0).

Digital Output pins:

HW Connection: Enabled.
Drive Mode: Strong Drive.

Initial State: High (1).

\section{BLOCK DIAGRAM AND SCHEMATICS}

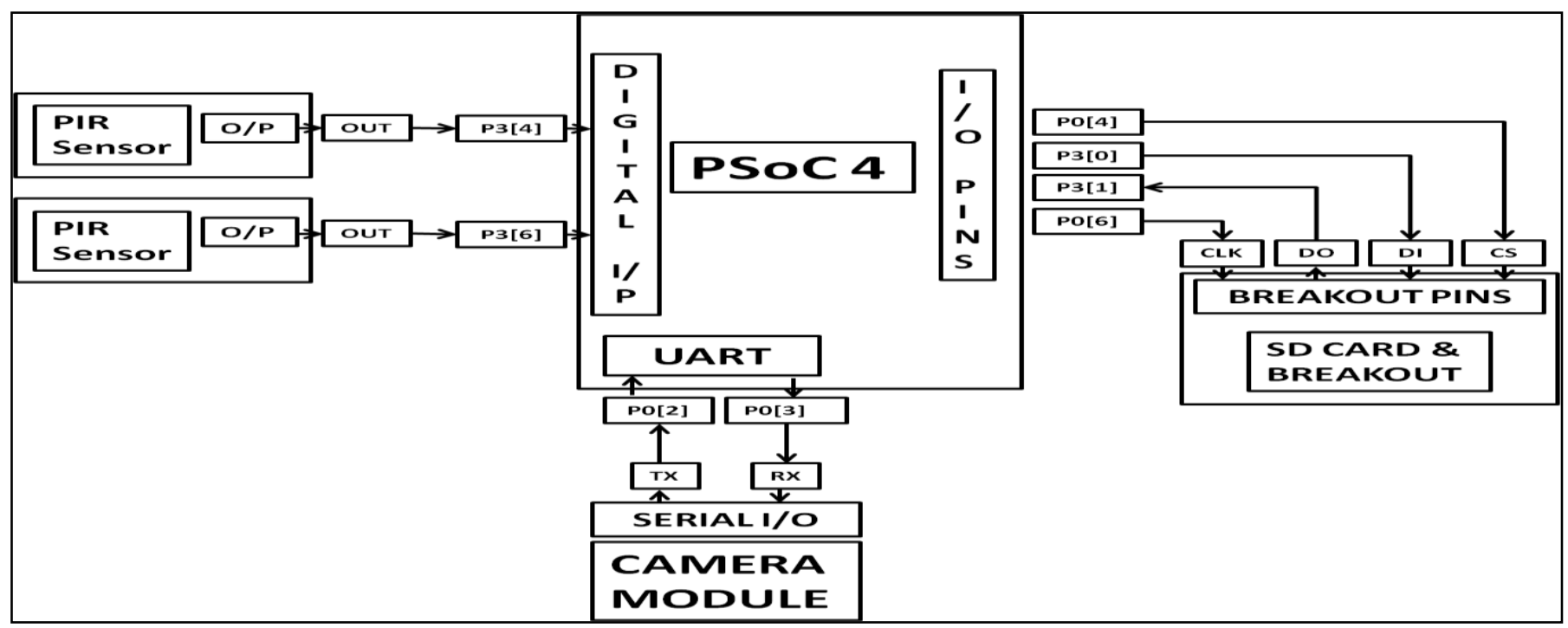

Fig 4: Block diagram showing the system functioning.

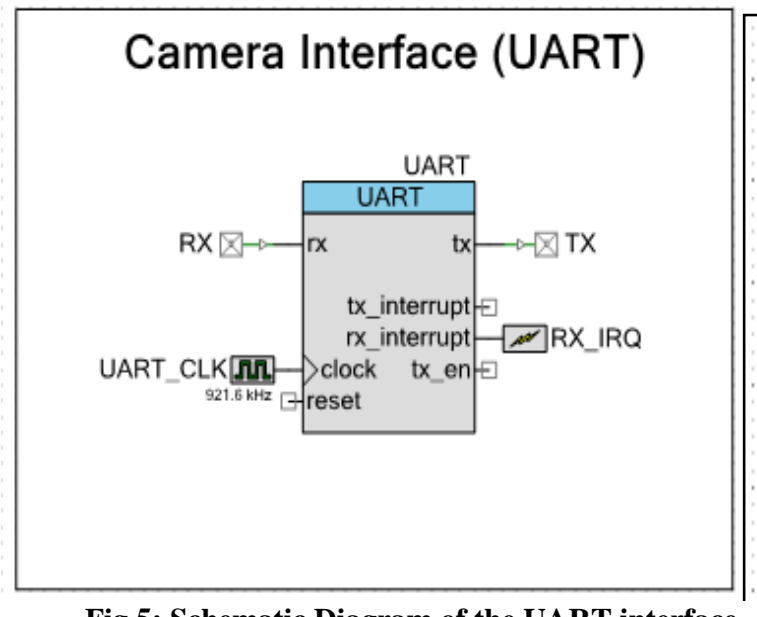

Fig 5: Schematic Diagram of the UART interface

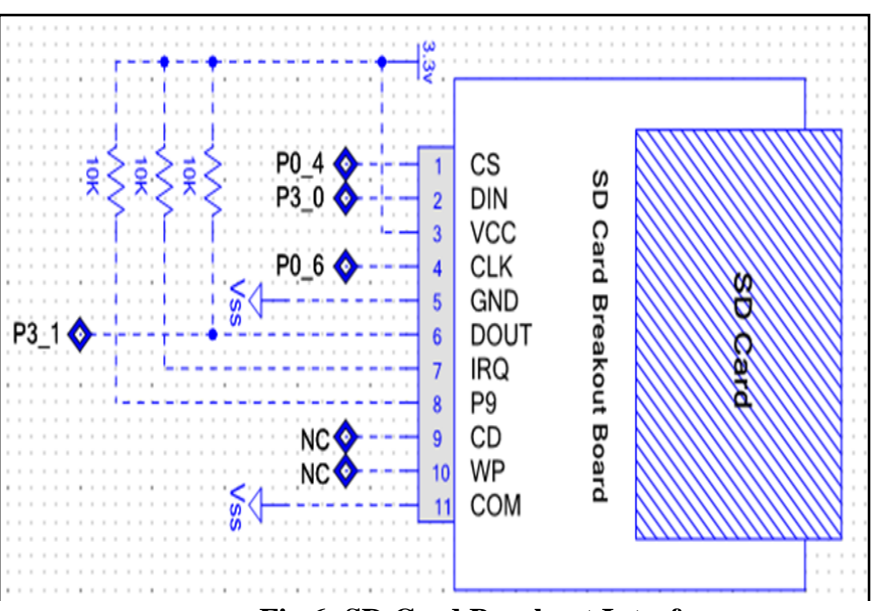

Fig 6: SD Card Breakout Interface

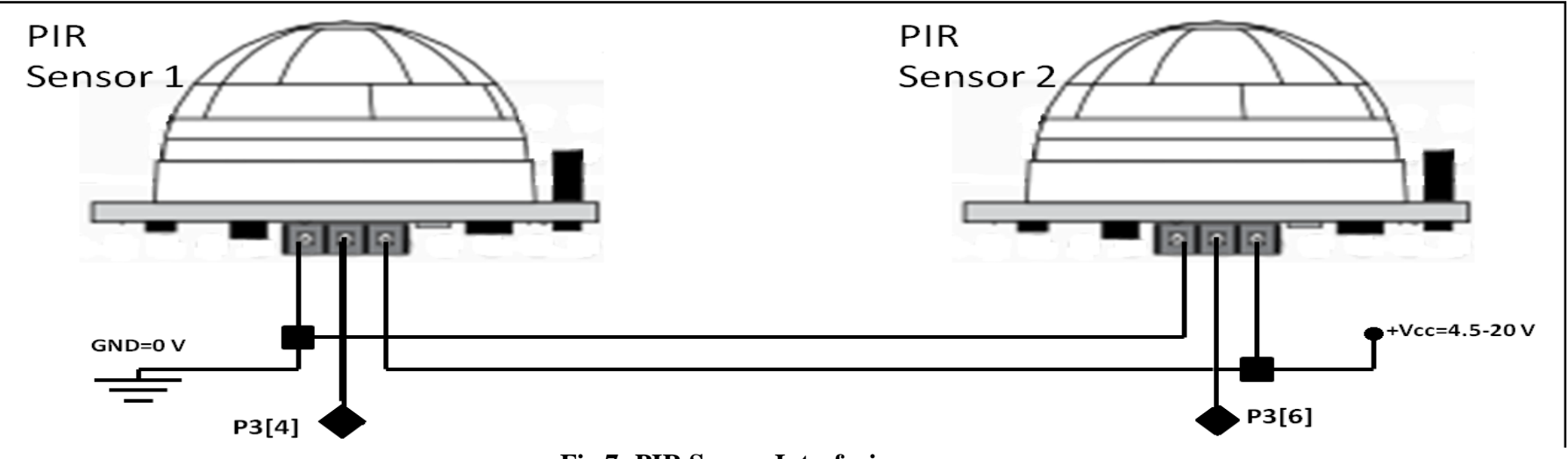

Fig 7: PIR Sensor Interfacing

\section{ALGORITHM TO DETECT SIGNAL JUMPING}

Note: This condition is only applicable when the signal turns red.
Step 1: Enable global interrupts.

Step 2: Initialize UART component of PSoC 4.

Step 3: Reset and synchronize the camera module.

Step 4: Initialize the SD card. 
Step 5: Initialize the camera module to store a JPEG image of resolution $640 \times 480$ pixels.

Step 6: Set the package size.

Step 7: Check for an active high signal from the PIR sensor, using only the first PIR sensor for this purpose. If a signal is received (indicating motion), execute steps 8 to 12 to capture a picture of the vehicle's license plates, else go to step 13 .

Step 8: Open a new file in the SD card to store an image.

Step 9: Program the Camera to take a JPEG snapshot and store it in the module's RAM.

Step 10: Send a command to the camera module to retrieve the image data from it. Calculate the length of data based on the acknowledgement received.

Step 11: The PSoC will receive in the form of several packages; each assigned a package ID that varies from 0x0000 to $0 \mathrm{xF} 0 \mathrm{~F} 0$.

Step 12: The image data is transferred from the PSoC to the SD card, storing it for later use.

Step 13: In case no activity is detected by the camera module for more than 15 seconds, it is synchronized again to prevent shutdown (The camera switches off if it is inactive for more than 15 seconds).

Step 14: Manual intervention is then required to access the SD card and view any images which can then be used to print a photo traffic ticket.

\section{ALGORITHM TO DETECT OVERSPEEDING}

Most of the steps of the above algorithm remain unaltered, except for the seventh step which is replaced by a number of steps as follows, and that this algorithm is only applicable during a green signal:

Step 7(i): When the first PIR sensor is activated by a moving vehicle, start incrementing a counter variable, which will stop when the second PIR sensor is triggered. If the counter drops below a certain threshold, it implies that overspending has taken place, and the steps 8 to 12 are executed as mentioned previously.

Step 7(ii): Stop incrementing the counter variable as soon as the second sensor is triggered.

Step 7(iii): Check the resulting counter value. If it is below a certain threshold, it implies that overspeeding has taken place, and steps 8 to 12 are executed as mentioned previously to take a picture of the vehicle's license plates, else go to step 13 .

\section{APPLICATIONS AND USEFULNESS}

As discussed earlier, the project can be use to catch signaljumping and over-speeding motorists. It can hence be used to enforce stricter traffic laws and serve as an effective deterrent to other motorists. The benefits could be seen as fewer traffic jams and better safety for pedestrians and other motorists. It can also be used to capture pictures of intruders and trespassers who trigger the PIR sensor, as evidence to be used against them in a court of law. [10]

It can be used for wildlife conservation, wherein a system of this sort captures pictures of animals which trigger the motion sensor. Taking pictures of endangered animals in this way is vital to monitoring species populations. [11]

The SD card output ensures that the evidence captured can be stored and accessed with ease at a later time, either by using an SD card reader or a device which accepts SD cards, such as a mobile phone or PC.

\section{LIMITATIONS}

This project can, as of now, only deal with one vehicle at a time, since the algorithm is unable to differentiate between various vehicles.
The system is not fully automated, as we require manual intervention at the last stage to extract the image from the SD card and print it out onto a photo traffic ticket, as well as identify the vehicle based on its license plates

The PIR sensor requires the vehicle to have a temperature different from that of its surroundings. This might not be detectable in cold climates.

\section{CONCLUSION}

This project on which this paper is based, has thus implemented an embedded system which can be, with a few modifications, successfully implemented in real time at traffic signals to catch motorists who jump signals and also to catch motorists who exceed the speed limits in other instances.

The external components used for the embedded system were studied. The configuration of the on-board PSoC components and the platforms used were examined as well. The algorithms, limitations and applications of the project were described at length.

The implementation of this system will thus reduce road accidents overall, leading to better safety on roads for both motorists and pedestrians, which is the aim of the project.

While still in its infancy, the project has various potential applications even outside the field of traffic law enforcement. The low power requirements enable it to be used with a battery power supply, and the low cost enables one to use the project for purposes where the budget is an issue. The aforementioned advantages allow the usage of the project for purposes ranging from wildlife conservation to intruder detection.

\section{REFERENCES}

[1] Sanjay Banerjee. "Traffic signal jumping soars to a new high", The Times of India, July 26, 2014

[2] Retting, Richard A., Robert G. Ulmer, and Allan F. Williams. "Prevalence and characteristics of red light running crashes in the United States." Accident Analysis \& Prevention 31.6 (1999): 687-694.

[3] 4D Systems. "Serial Camera Module uCAM-II datasheet."

[4] Marlin P. Jones \& Association. "HC-SR501 PIR MOTION DETECTOR"

[5] PSoC ${ }^{\circledR}$ Creator ${ }^{\mathrm{TM}}$ Component Datasheet. "SDCard 1.0."

[6] Ashby, Robert. "Designer's guide to the Cypress PSoC." Vol. 10. Newnes, 2005.

[7] Programmable System-on-Chip (PSoC®) datasheet. "PSoC® 4: PSoC 4200 Family."

[8] PSoC® Creator ${ }^{\mathrm{TM}}$ Component Datasheet. "Universal Asynchronous Receiver Transmitter (UART) 2.30"

[9] PSoC® Creator ${ }^{\mathrm{TM}}$ Component Datasheet. "Pins 1.90"

[10] Moghavvemi, M.; Lu Chin Seng, "Pyroelectric infrared sensor for intruder detection," TENCON 2004. 2004 IEEE Region 10 Conference, Vol. D, No., pp.656,659, Vol. 4, 21-24 Nov. 2004, DOI: 10.1109/TENCON.2004.1415018

[11] Brown, Justin, and Stanley D. Gehrt. "The basics of using remote cameras to monitor wildlife." Ohio State University Extension Agriculture and Natural Resources Fact Sheet W-21-09. Ohio State University, Columbus, $\mathrm{OH}(2009)$. 